\title{
A New Method of Video Recording the Posterior Fundus Segment
}

\author{
DOI: $10.17691 / \mathrm{stm} 2019.11 .2 .19$
}

Received December 12, 2018

D. Doroshenko, Head Physician';

V.K. Salakhutdinov, PhD, Leading Researcher';

Yu.G. Smetanin, DSc, Chief Researcher ${ }^{3}$;

G.E. Stolyarenko, MD, DSc, Professor, General Director ${ }^{1}$;

M.Yu. Ledeneva, MD, DSc, Physician ${ }^{1}$

${ }^{1}$ LLC "Center for Diagnosis and Surgery of Posterior Eye Segment", 2, 2nd Vladimirskaya St.,

Moscow, 111123, Russia;

${ }^{2}$ Federal Research Center Scientific Research Institute for System Studies of the Russian Academy of Sciences

Bldg 1, 36 Nakhimovskiy Prospekt, Moscow, 117218, Russia;

${ }^{3}$ Federal Research Center "Informatics and Management" of the Russian Academy of Sciences, Bldg 2 ,

44 Vavilova St., Moscow, 119333, Russia

The paper presents the results of developing a new technology for recording the images of posterior fundus tissues, which allows us to improve the visibility of information-significant objects reducing the light load on the retinal tissue. Considerable attention is paid to the issue of increasing the robustness of the recorded image in low light conditions.

Key words: the retina; illumination of the surgical field on the retina; vitreoretinal surgery.

\section{Introduction}

Insufficient illumination of the surgical field is a challenging problem of vitreoretinal surgery [1]. This problem is particularly acute in videographing and TV-broadcasting of the vitreoretinal surgery process for educational purposes and in telemedicine, when requirements for TV-image quality are high [2].

The modern illuminator of the fundus is a powerful source of light with the spectrum close to that of the sunlight, its radiation emitted through an optical fiber and a hole in the sclera into the eye cavity. The issue is complicated as the reserves for improving the operating field illumination only by increasing the intensity of light transmitted to the eye cavity are practically exhausted. That is caused by the fact that the light power density provided by modern illuminators on the retinal surface is nearly maximum allowed for avoiding light damage to the retina and in some cases it exceeds $1 \mathrm{~mW} / \mathrm{mm}^{2}$ $[3,4]$.

The problem of excessive exposure of the retina to radiation turns into an insoluble one in case of using OCT intraoperatively when the retina is exposed to radiation in the near-IR range along with the fundus tissue illumination.

The paper presents the results of developing a new technology for recording the image of posterior fundus tissues. Considerable attention is paid to the issue of increasing the robustness of the recorded image in low light conditions.

\section{Materials and Methods}

The main idea is to use the illuminator as a set of several monochromatic light sources with different wavelengths. High robustness of recording the information significant features is achieved by optimizing the wavelengths of radiation from these sources and software processing of images obtained by illuminating the operating field with each individual monochromatic light source. The ratio of visibility to the light power density on the illuminated surface is taken as efficiency criterion.

Figure 1 shows a block diagram of a spectral-selective illuminator. The device consists of several laser light sources $\left(L s_{1}, L s_{2}, \ldots, L s_{n}\right)$, each emitting at a specific wavelength $\left(\lambda_{1}, \lambda_{2}, \ldots, \lambda_{n}\right)$; a multiplexer (M) combining the radiation of all lasers and integrating it in a single multimode optical fiber (MOF); the element of coherent artifact suppression (CAS) and laser light source control driver; a monochrome camera and a video grabber that provide capturing a color image with high light sensitivity and high resolution [5].

Radiation from each source is fed through a separate

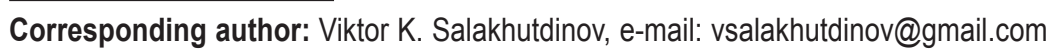




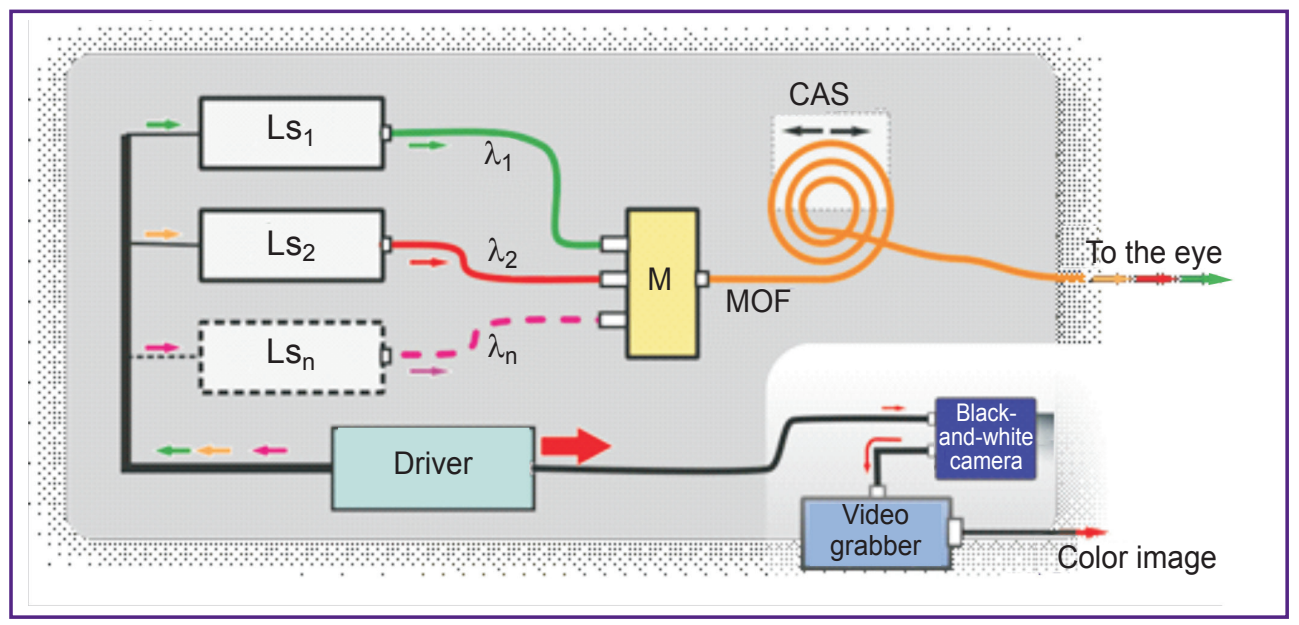

Figure 1. Block diagram of spectral-selective illuminator

See the text for explanations

optical fiber to the mixer. Multiplexer is used to combine and carry radiation from all sources into a multimode fiber through which light is transmitted to illuminator output. High spatial homogeneity of the output beam is achieved through the use of CAS. The high-speed video camera captures a black-and-white image, which is converted to a color one according to the algorithm described below.

Spectrum optimization. The method efficiency is determined by the spectral composition of the radiation. It is easy to notice that, in general, the problem of illuminator spectrum optimization is reduced to the classical task of detection, estimation, and modulation [6] as described in terms of solution theory. The problem is that in our case, the number of information significant objects $(N)$ significantly exceeds the number of spectral components in illuminator emission $(m)$ and it is impossible to obtain light with an arbitrary wavelength $(\lambda)$ using lasers.

Therefore, methods and approaches of detection and estimation theory based on optimal detection criteria of Neumann-Pearson are hardly suitable for solving our problem. However, in this case, constrained problem can be reduced to partition of graph describing the relationship between objects:

$$
N(i, j)=\sum_{k=1}^{m(i)} N_{1}(i, k) N_{2}(k, j)=\sum_{k=1}^{m(i)}\left|E_{i}^{k} \cap E^{j}\right|=\left|\bigcup_{k=1}^{m(i)} E_{i}^{k} \cap E^{j}\right|,
$$

where $N$ is a matrix of element relations; $N_{1}$ is a block matrix showing which elements $k$ are the result of partition $i ; m(i)$ is the number of such elements; $N_{2}$ is a matrix of element relations $k$ with unseparated elements $j ; E$ is a set of partitions.

The greedy algorithm was used for numerical calculations. Spectra of information-significant objects were taken from the works [7-11], while those of the applied laser sources were from the available websites [12]. Numerical simulation has shown that with no regard for national and racial characteristics of pigmentation, visualization of the retinal blood vessel topology and its differentiation according to oxygenation degree are achieved by using an illuminator consisting of only two lasers with wavelengths $\lambda_{1}=577 \mathrm{~nm}$ and $\lambda_{2}=650 \mathrm{~nm}$. At the same time, adding a third light source with $\lambda_{3}=520 \mathrm{~nm}$ enables additional and energetically effective visualization of the vitreous humor, the posterior hyaloid membrane, retinal detachment, and pigment epithelial defects, while reducing the light power density required for visually comparable image quality twice.

Video recording particulars. A color video camera can only capture the image of the operating field with unacceptably low quality due to the reduced total energy of light striking the

Figure 2. Video recording algorithm 
Figure 3. Video recording frames of the operating field on the retina, obtained using different light sources:

(a) with xenon illuminator; (b), (d) with spectralselective illuminator $(\lambda=520,577$, and $650 \mathrm{~nm})$ and (c) with LED illuminator. The arrow points to stained posterior hyaloid membrane
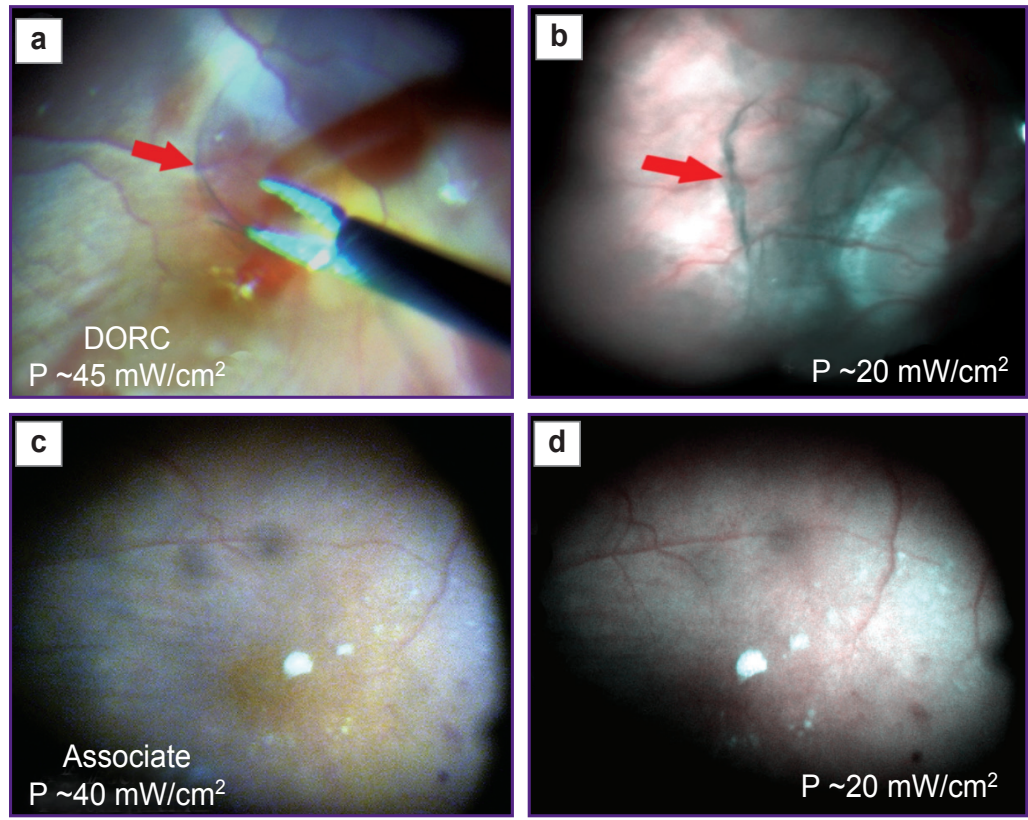

retina. This is attributable to insufficient light sensitivity of color cameras. Since black-and-white video camera sensitivity is remarkably greater than that of the color one, we applied a high-speed black-and-white camera for video recording of color images. Figure 2 presents an algorithm that allows to capture a color image using a black-and-white camera and a spectral-selective illuminator with several (in our case, three) light sources.

The light sources are switched on sequentially, each for the time of recording a single frame with the black-and-white camera. Since each source emits monochromatic light, each black-and-white camera frame captures only one color component of the object being recorded. In such a case, the sequence of blackand-white frames $n$ (where $n$ is the number of light sources) is a necessary and sufficient set of informationsignificant color components of the color image of the recorded object. The video grabber buffers a sequence of black-and-white frames $n$ and reconstructs one frame of the color image of the recorded object using this sequence.

Experimental result. The results of numerical simulation were verified in a medical experiment. The spectral-selective illuminator used lasers with wavelengths of 520,577 , and $650 \mathrm{~nm}$ as light sources. Here we present the results of video recording of the operating field on the retina using a xenon illuminator (Figure $3(\mathrm{a})$ ), spectral-selective illuminator DORC Xenon BrightStar (Figure 3 (b), (d)), and LED illuminator Associate (Figure 3 (c)) (DORC, Netherlands).

Frames (a) and (c) were obtained using color CCDvideo camera B1641 at light power density in the arrow region equaling $40-45 \mathrm{~mW} / \mathrm{cm}^{2}$ and frequency of 30 frames per second. Frames (b) and (d) were obtained using a black-and-white CMOS-camera DMK
$33 G \times 236$ at light power density in the central region of the frame $\approx 20 \mathrm{~mW} / \mathrm{cm}^{2}$ and frequency of 50-60 frames per second. The algorithm described in [5] was used to convert black-and-white image frames into color picture. Power ratio of individual light sources in the spectral-selective illuminator and weight functions of black-and-white video stream processing algorithm [5] were selected based on the criterion of maximum visual correspondence with the image obtained using LED illuminator Associate.

Figure 3 (a) and (b) demonstrate the quality of posterior hyaloid membrane image visualization in membranectomy. It can be seen that in spectral-selective lighting the contrast and resolution are higher, while light load on the retinal tissue is significantly less.

Figure 3 (c) and (d) demonstrate the quality of retinal and choroid topology visualization. It can be seen that in spectral-selective lighting the image conveys the details of the retinal topology and choroid defects better. This is largely attributable to considerable integral light penetration depth, elimination of transverse chromatic aberrations at the stage of converting a black-and-white image into a color one and robustness of video recording to reduced illumination.

\section{Conclusion}

The use of a spectral-selective illuminator as a light source increases image contrast while reducing the light load on the retina. Only three commercially available low-cost lasers are required to generate emission of the spectral-selective source. Reducing the light load provides the possibility to apply additional diagnostic methods based on the use of light, such as endo-OCT, intraoperatively. 
Another advantage of the proposed method is increasing the robustness of video recording in low light conditions.

Study funding. This study was partially supported by funding from the Russian Foundation for Basic Research within the framework of scientific projects No.18-0700882, No.17-07-00561, and No.19-07-00395 in part of software implementation of quantification algorithm.

Conflict of interests. The authors have no conflict of interests to disclose.

\section{References}

1. Sakaguchi H., Oshima Y. Considering the illumination choices in vitreoretinal surgery: continual improvements allow for better, safer outcomes. Retin Physician 2012; 3: 26-31.

2. Spectral domain optical coherence tomography in macular diseases. Meyer C.H., Saxena S., Sadda S.R. (editors). New Delhi: Springer; 2017.

3. Charles S. Illumination and phototoxicity issues in vitreoretinal surgery. Retina 2008; 28(1): 1-4, https://doi. org/10.1097/IAE.0b013e318156e015.

4. Yamgutdinov R.R., Mukhamadeev T.R., Yamlikhanov A.G.,
Dibaev T.I., Aznabaev B.M. Brightness and phototoxicity two sides of endoillumination. Meditsinskiy vestnik Bashkortostana 2017; 13(1): 127-131.

5. Milen'kaya T.M., Salakhutdinov V.K. Fundus camera. Patent RU 2215463. 2003.

6. Harry L. Van Trees. Detection, estimation, and modulation theory. Part I. New York: John Wiley and Sons; 1968.

7. Tabulated molar extinction coefficient for hemoglobin in water. URL: https://omlc.org/spectra/hemoglobin/summary. html.

8. Schmitt J.M. Optical measurement of blood oxygenation by implantable telemetry. Technical Report G558-15. Stanford; 1986.

9. Moaveni M.K. A multiple scattering field theory applied to whole blood. Ph.D. Dissertation. Dept. of Electrical Engineering, University of Washington; 1970.

10. Hammer M., Schweitzer D. Quantitative reflection spectroscopy at the human ocular fundus. Phys Med Biol 2002; 47(2): 179-191, https://doi.org/10.1088/00319155/47/2/301.

11. Delori F.C., Pflibsen K.P. Spectral reflectance of the human ocular fundus. Appl Opt 1989; 28(6): 1061, https://doi. org/10.1364/ao.28.001061.

12. Changchun New Industries Optoelectronics Technology Co., Ltd. Lasers, Optics \& Photonics. URL: http:// www.cnilaser.com. 